\title{
Guided resonance reflective phase shifters
}

\author{
Yu Horie, Amir Arbabi, and Andrei Faraon \\ T. J. Watson Laboratory of Applied Physics, California Institute of Technology, \\ 1200 E. California Blvd., Pasadena, CA 91125, USA
}

\begin{abstract}
We present reflective phase shifters based on high contrast gratings resting on a low-index spacer backed by a metallic mirror. The guided resonance of the grating combined with the reflection from the metallic mirror leads to an all-pass filter with $2 \pi$ phase shift variation and unity reflectivity across the resonance. We present simulations, fabrication and measurement of passive devices fabricated in silicon over gold using a polymer as the spacer layer. Active control at high modulation speeds can be achieved by shifting the guided resonance wavelength using carrier injection or thermo-optic effect in silicon.
\end{abstract}

Keywords: Spatial light modulator, phase modulator, all-pass filter, guided resonance, sub-wavelength grating, high-contrast grating, high index contrast, metasurface

\section{INTRODUCTION}

Spatio-temporal control of the phase of light has attracted much attention in many fields of optics including beam steering, adaptive optics, microscopy, Fourier optics, and holography. ${ }^{1}$ Reflective phase-only spatial light modulators (SLMs), which are composed of arrays of all-pass phase modulators, are widely used for on demand modification of wavefronts of optical beams. Commercially available phase modulators rely on liquid crystal (LC) technology, but the relatively slow response of LCs limits the modulation speed of such phase modulators to a few $\mathrm{kHz} .^{2}$ Micro-electro-mechanical system (MEMS) modulators offer higher modulation speeds (typically up to one $\mathrm{MHz}$ ), but their complicated fabrication processes make them less attractive for mass production. Recently, MEMS tunable all-pass filters have been demonstrated which use high-contrast grating (HCG) reflectors in a Gires-Tournois interferometer (GTI) configuration. ${ }^{3,4}$ The light weight of the HCG reflectors has increased the modulation bandwidths of these devices to over $500 \mathrm{kHz}$. Here, we propose and experimentally demonstrate phase shifters composed of sub-wavelength high contrast gratings resting on a low-index spacer layer which is backed by a metallic mirror. The guided resonance of the grating combined with the reflection from the metallic mirror lead to an all-pass filter with $2 \pi$ phase shift variation and unity reflectance across the resonance. Rigorous coupled-wave analysis (RCWA) technique was used to verify the phase shifting scheme. We present fabrication process and measurement procedure of all pass reflective filters operating at telecom wavelengths. These filters are realized by fabricating silicon HCGs on a polymer spacer layer over gold. Active control with high modulation bandwidths can be achieved by tuning the guided resonance frequency using carrier injection or thermo-optic effect that change the Si's refractive index. Integration of an array of such phase shifters will lead to reflective phase-only SLMs operating at faster speed than state-of-the-art devices.

Further author information: (Send correspondence to A.F.) A.F.: E-mail: faraon@caltech.edu, Telephone: 16263953086

High Contrast Metastructures IV, edited by Connie J. Chang-Hasnain, David Fattal,

Fumio Koyama, Weimin Zhou, Proc. of SPIE Vol. 9372, 93720W · @ 2015 SPIE

CCC code: $0277-786 \mathrm{X} / 15 / \$ 18 \cdot$ doi: $10.1117 / 12.2077744$

Proc. of SPIE Vol. $937293720 \mathrm{~W}-1$ 


\section{METHOD AND RESULTS}

\subsection{Design of all-pass phase shifters}

A schematic diagram of the proposed reflective phase shifter is depicted in Fig. 1(a). The reflective phase shifter consists of a sub-wavelength grating and a metallic reflector separated by a spacer layer. The sub-wavelength grating is designed to support a guided resonance, ${ }^{5,6}$ which can be excited by a slightly tilted off-normal plane wave with a TE polarization in the telecom wavelength around $1.55 \mu \mathrm{m}$. By placing a metallic reflector more than a wavelength apart under the grating, it is expected that reflected amplitude becomes constant regardless of the guided resonance, while the reflection phase shift is still dispersive with a $2 \pi$ phase variation, due to the coupling into the guided resonance of the grating. As schematically plotted in Fig. 1(b), the modulation of the phase while keeping the reflection power constant can be done by tuning the wavelength of the guided resonance by refractive index tuning of the grating materials. ${ }^{7}$ For instance, in the case of Si gratings, carrier injection ${ }^{8}$ or thermo-optic effect can be employed to efficiently tune the resonance wavelength. Using RCWA method, reflection spectra as well as the phase shift for the reflected wave as a function of wavelength are calculated for

(a)

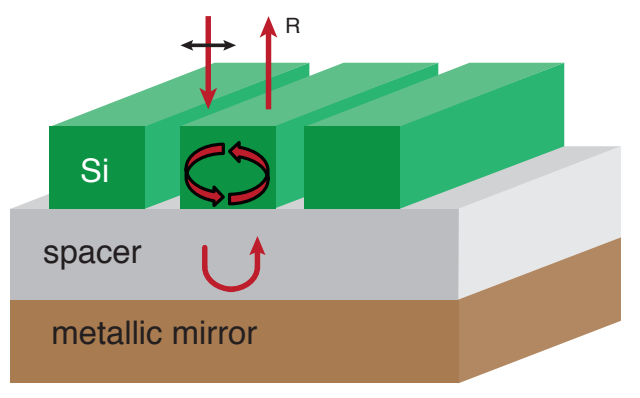

(c)

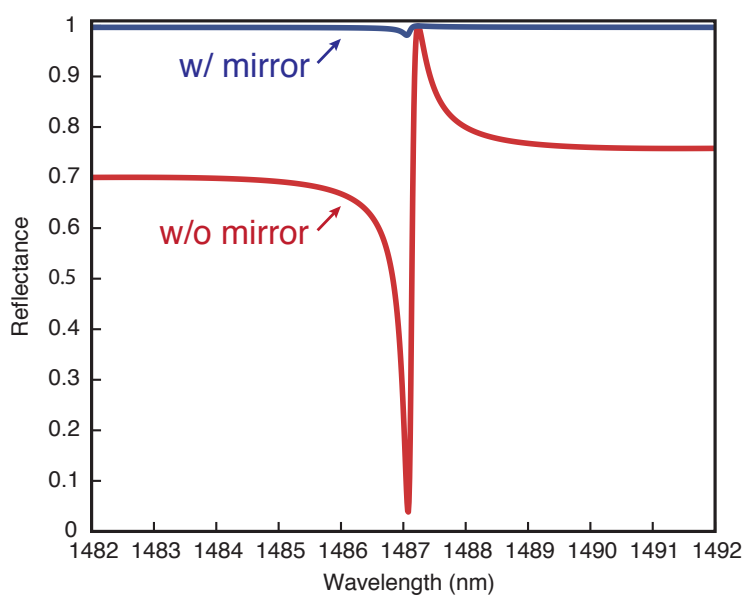

(b)

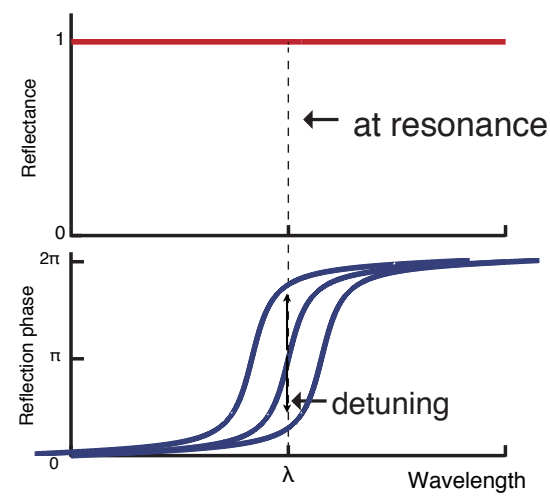

(d)

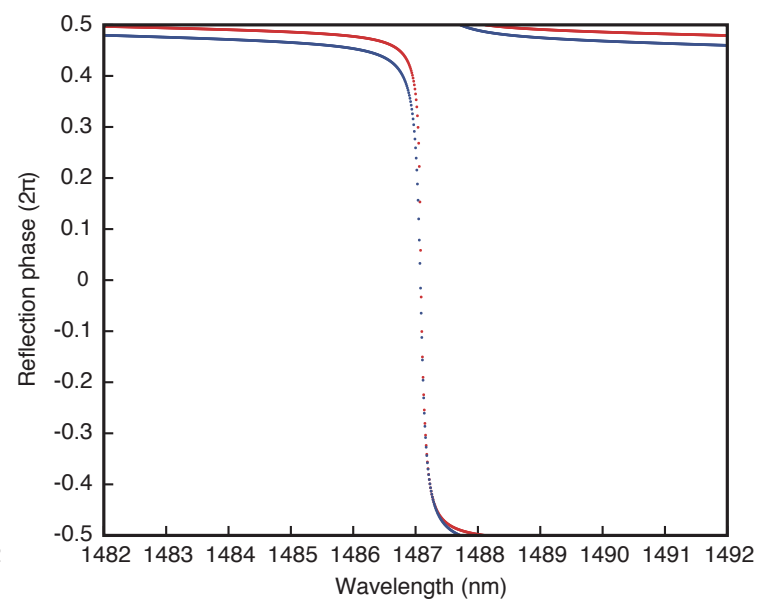

Figure 1. (a) Schematic illustration of the proposed reflective phase shifters. (b) Operating principle of phase modulation using all-pass filters. (c) Reflection spectra and (d) reflection phase shift with and without a reflector for the reflective phase shifters. 
two cases of with and without the backside reflector, and are shown in Fig. 1(c,d). Without the metallic mirror underneath, the reflection spectrum around the guided resonance (plotted in red) exhibits the Fano line shape. ${ }^{6}$ On the other hand, when the grating is placed above a reflective metal with a spacer layer made of $3 \mu$ m-thick $\mathrm{SiO}_{2}$ separating the two, almost unity reflectance is observed, while the $2 \pi$ phase shift for the reflected wave is preserved.

To investigate the feasibility of phase modulation by refractive index tuning, a set of finite-difference timedomain (FDTD) simulations were performed. In the FDTD simulations, a continuous wave source was used to excite the guided resonance in the grating, and the reflected phase values were obtained as we changed the refractive index of the Si grating bars. Figure 2 shows that the phase of the reflected wave is gradually modified with the refractive index change of $\Delta n \sim 4 \times 10^{-3}$ in $\mathrm{Si}$, covering the whole linewidth of the guided resonance with a quality factor of $2.5 \times 10^{3}$. This amount of index tuning can be easily obtained by thermo-optic effect in $\mathrm{Si}$, or even by carrier injection method. Considering the optical loss due to the free carrier absorption effect, the moderate target quality factor for the guided mode should be a few thousands. Using the RCWA method including the free carrier absorption effect, $50 \%$ of the optical power would be lost when the guided resonance is designed to have a quality factor of $10^{4}$, and $20 \%$ absorption loss is expected with a quality factor of $5 \times 10^{3}$.

\subsection{Fabrication}

The fabrication process for the all-pass reflective filters composed of sub-wavelength gratings backed by a metallic reflector is illustrated in Fig. 3. We start from two substrates: a silicon-on-insulator (SOI) and a Si substrate. The grating pattern was defined by electron beam lithography using a positive resist (ZEP-520A) followed by inductively coupled plasma reactive ion etching (ICP-RIE) process using $\mathrm{SF}_{6} / \mathrm{C}_{4} \mathrm{~F}_{8}$ chemistry for patterning the Si layer. An $80 \mathrm{~nm}$-thick gold layer was deposited on the Si substrate. $2 \mu$ m-thick SU-8 polymer was spun on the both substrates, followed by a 2 minute pre-bake at $95^{\circ} \mathrm{C}$ to partially evaporate its solvent. Then, the two substrates were manually brought into contact and loaded into a vacuum chamber. A uniform pressure was applied to the chips at $95^{\circ} \mathrm{C}$ to ensure the SU-8 reflow. After 20 hours of adhesive bonding process, the donor substrate for the SOI side was removed by using an $\mathrm{SF}_{6} / \mathrm{C}_{4} \mathrm{~F}_{8}$-based deep reactive ion etching (DRIE) process. After removing the substrate from the SOI side, the adhesive SU-8 bonding layer was exposed with an

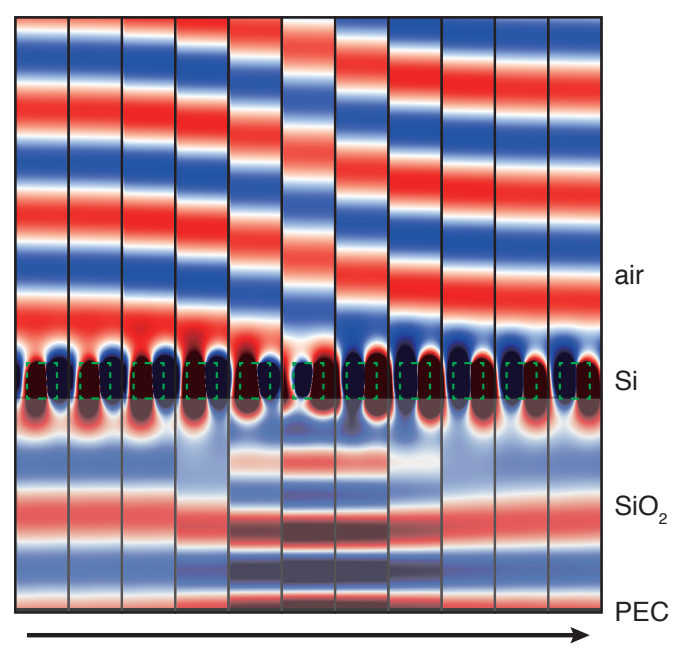

$\Delta \mathrm{n}(\mathrm{Si})=4 \times 10^{-3}$

Figure 2. A sequence of snapshots of the electric field reflected from the all-pass filter as the Si refractive index is tuned. The FDTD simulations are performed with continuous wave excitations. 


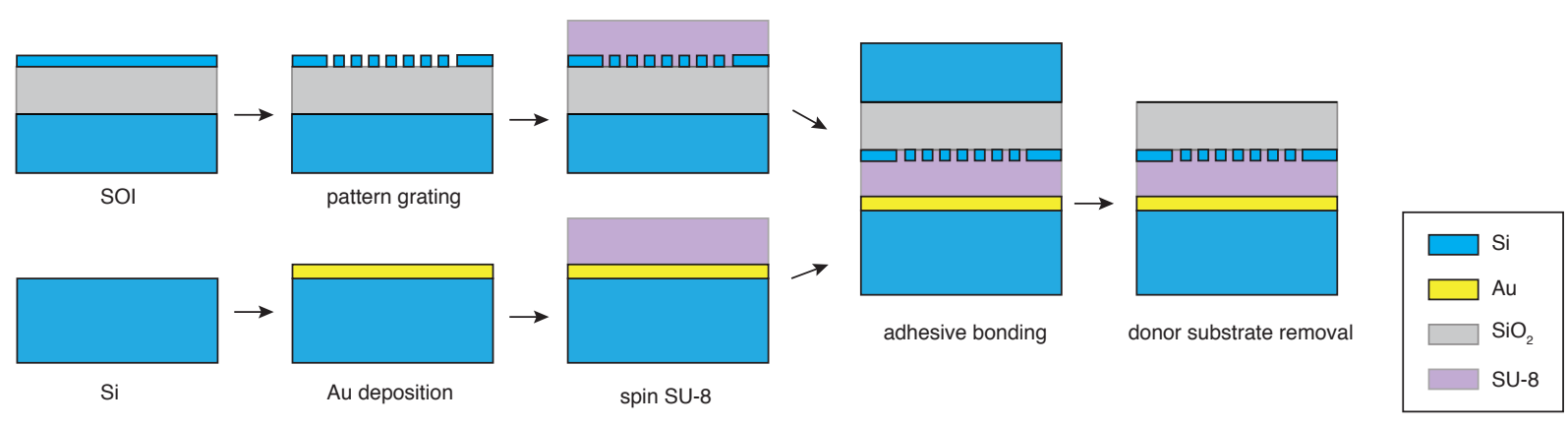

Figure 3. Fabrication process for reflective phase shifters. The adhesive bonding process followed by a donor substrate removal is used to transfer the $\mathrm{Si}$ grating pattern onto the $\mathrm{Au} / \mathrm{Si}$ substrate.

i-line irradiation and post-baked at $95^{\circ} \mathrm{C}$ for the purpose of cross-linking. Finally the SU-8 layer was cured by hard-baking at $200^{\circ} \mathrm{C}$ for 30 minutes.

\subsection{Phase measurement for all-pass reflective filters}

The fabricated all-pass filters were characterized by measuring their reflection spectra as well as reflection phase. The reflection spectra were measured by scanning the wavelengths of a tunable laser and collecting the reflected light intensity from the grating using an InGaAs photodetector. The reflection spectra of a device for TE and TM incidence are plotted in Fig. 4(b). Only the TE polarized light can couple to the resonance of interest in the fabricated devices. The quality factor of this resonance is $\sim 2 \times 10^{2}$. To evaluate the reflection phase, we used the cross-polarization optical setup schematically shown in Fig. 4(a), in which the reflection phase information can be deduced from the detected signal as a result of interference of the resonator-coupled light with a reference beam of known amplitude and phase. In this case, the TM-polarized light is the reference beam which is interfered with the resonator-coupled TE-polarized light. After passing the laser light through a polarizing beam splitter (PBS), it passes a quarter wave plate (QWP) which is placed in front of the devices to create an elliptically polarized beam. Using the Jones matrix method, the detected signal after the PBS resulted from the interference of the two beams can be written as

$$
\begin{aligned}
I_{\text {out }}(\lambda) & =\left|E_{\text {out }}\right|^{2}=|\langle H|Q(\theta) S(\lambda) Q(\theta)| V\rangle|^{2} \\
& =A(\theta)\left(r(\lambda)+e^{i \Psi(\theta)}\right),
\end{aligned}
$$

where $r(\lambda)$ is the complex reflection coefficient of the device, $Q(\theta)$ and $S(\lambda)$ are the Jones matrices of the QWP and the resonator, respectively,

$$
\begin{aligned}
A(\theta) & \equiv \frac{i}{2}\left(\cos ^{2}(2 \theta)-i \sin (2 \theta)\right) \\
e^{i \Psi(\theta)} & \equiv \frac{\cos ^{2}(2 \theta)+i \sin (2 \theta)}{\cos ^{2}(2 \theta)-i \sin (2 \theta)}
\end{aligned}
$$

and $\theta$ is the QWP angle. A series of spectra obtained by changing the QWP angle $\theta$ was collected. The complex amplitude of the reflection coefficient $r(\lambda)$ was obtained by fitting the measured spectra to the theoretical model using a least square method, and the reflection phase spectrum was computed by taking the angle of the reflection coefficient. The computed reflection phase as a function of wavelength is shown in Fig.4(c). The computed reflection phase varies by more than $\sim 3 \pi / 2$ around the resonance.

\section{CONCLUSION}

We proposed reflective phase shifters based on sub-wavelength gratings with guided resonances resting on a low-index spacer backed by a metallic mirror. The feasibility of the active control for phase modulation was 
(a)
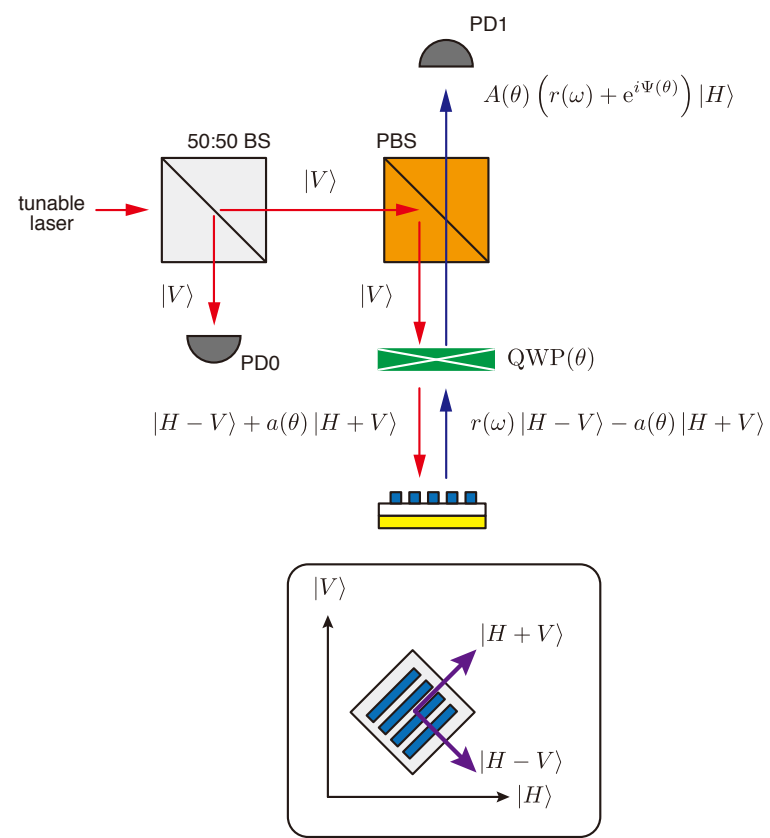

(b)

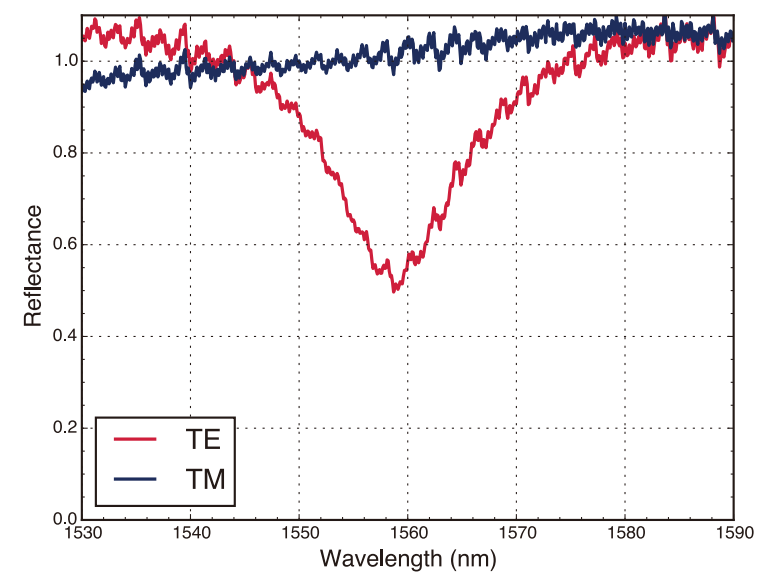

(c)

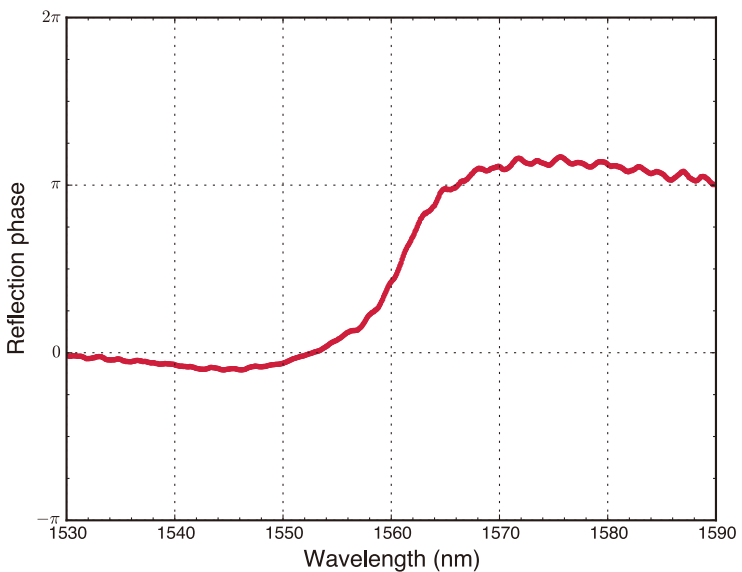

Figure 4. (a) Cross-polarization setup for the phase measurements. (b) Reflection spectra for the resonance with TE and TM incidence. (c) Computed reflection phase as a function of wavelength.

studied using numerical simulations. The passive reflective phase shifters were fabricated in silicon over gold using a polymer as the spacer layer and were characterized. Active modulation of these all-pass filters by shifting their guided resonance wavelengths using carrier injection or thermo-optic effect in silicon provides a promising approach for achieving high bandwidth spatial light modulators. Structures with higher quality factors will be fabricated to allow for efficient modulation.

\section{REFERENCES}

1. Efron, U., [Spatial Light Modulator Technology: Materials, Devices, and Applications], CRC Press, New York (1994).

2. Igasaki, Y., Li, F., Yoshida, N., Toyoda, H., Inoue, T., Mukohzaka, N., Kobayashi, Y., and Hara, T., "High efficiency electrically-addressable phase-only spatial light modulator," Optical Review 6(4), 339-344 (1999).

3. Yang, W., Sun, T., Rao, Y., Chan, T., Megens, M., Yoo, B.-W., Horsley, D. A., Wu, M. C., and ChangHasnain, C. J., "Optical phased array using high-contrast grating all-pass filters for fast beam steering," in [Proc. SPIE], 8633, 86330G (2013). 
4. Megens, M., Yoo, B. W., Chan, T., Yang, W., Sun, T., Chang-Hasnain, C. J., Wu, M. C., and Horsley, D. A., "High-contrast grating MEMS optical phase-shifters for two-dimensional free-space beam steering," in [Proc. SPIE], 8995, 89950Q (2014).

5. Fan, S. and Joannopoulos, J. D., "Analysis of guided resonances in photonic crystal slabs," Phys. Rev. B 65, $235112(2002)$.

6. Fan, S., Suh, W., and Joannopoulos, J. D., "Temporal coupled-mode theory for the Fano resonance in optical resonators.," J. Opt. Soc. Am. A: Opt. Image Sci. Vis. 20(3), 569-572 (2003).

7. Horie, Y., Arbabi, A., and Faraon, A., "Reflective Optical Phase Modulator Based on High-Contrast Grating Mirrors," in [CLEO: 2014], STh4M.8 (2014).

8. Qiu, C., Chen, J., Xia, Y., and Xu, Q., "Active dielectric antenna on chip for spatial light modulation," Sci. Rep. 2, 855 (2012). 